\title{
Design and Implementation of College Physical Education Management System
}

\author{
Mei-huan Zhao \\ \{zhaomeihuanzz@163.com\} \\ Department of tactics, Henan Police College Zhengzhou 450046, China
}

\begin{abstract}
Based on the current development situation of the cloud platform, this paper points out that the construction and application of enterprise management application platform in some small and medium enterprises is the main development trend of cloud services. Learn from their successful experience, aiming at the university sports educational management system, the application system design and implementation of cloud platform is studied based on the integration of cloud computing, based on a number of computer resources, designed the model of cloud application platform, greatly improves the computing and storage function management application function the platform, which cater to the individual needs more physical educational administrative staff, enhance the quality of college physical educational management, network service quality and work efficiency, not only assist sports management personnel in Colleges and universities can correctly and quickly get the most accurate information of sports and sports facilities, sports venues and other information cost information, also the management of school sports teaching departments efficiently complete the daily sports management work in the shortest time, and timely decision Policy.
\end{abstract}

Keywords: Cloud computing; MVC; cloud application; cloud storage; RIA; colleges and universities

\section{Introduction}

Since the formation of the concept of cloud computing, it has been very rapid development, especially by the development and research of Google Corporation and International Busine Machines Corp, for the future development of the network pointed out the direction. By means of by multiple computers with the integration of resources, by comprehensive type of computing power, on the basis of related infrastructure, software and hardware conditions can obtain storage, computing and many other services, the cloud computing basic definitions. When building a network service platform with cloud computing, the network resources can be effectively integrated, which will provide more diverse and highquality services to users. Users in the system platform can submit service application information, the use of some non local resources, he through the system program, in terms of maintenance and functional extensions with high performance. With the development and improvement of science and technology, the development and improvement of cloud computing has been developed rapidly [1], and the related technologies have been widely used. As using cloud computing technology, the core of the enterprise, Google give full play to the advantages of the technology, greatly reduce the operation cost of the system, has good 
storage and computing functions, great economic benefit has been obtained. In addition, Amazon Co also conducted in-depth exploration and research in terms of cloud computing technology, its independent research and development of simple storage services, Web Services - elastic computing cloud, cloud service platform makes the performance greatly improved. China is now finding status, based on the Internet provides software and services software service is the main form of cloud platform, including customer relationship management, enterprise resource planning system, online financial management software and eight hundred passengers, etc.. The formation and development of cloud computing has become the main development trend of network services, in this context, it is necessary to improve the service related to the large data and information. In modern society, the system needs to handle the information and data quantity is more and more big, this requires configuration of the corresponding hardware equipment, in order to achieve massive data unified processing, computing and storage, at the same time in order to meet the requirements of the safety performance of the system must be to increase the user's hardware investment. For smaller companies, hardware cost increases will directly affect the economic benefits, in addition to the need of software configuration, the renewal and the maintenance etc. a series of management, increasing the financial burden on users. In view of the above problems, in cloud computing, research and development of the network service platform, include a number of features and functions, to meet the business needs of small and medium enterprises, for enterprise's development and growth has created a good opportunity [2].

\section{Cloud Computing Theory and Its Key Technologies}

After long-term development and improvement, network storage, network computing, etc. a variety of advantageous resources are sufficient mining and development, which including education and technology R \& D industry has very important influence, the information data sharing and real-time communication for reality, work efficiency has been improved. In the network computing cost expenses also significantly reduced, has formed a distributed computing core technology, utility computing, Internet Computing and according to the required services such as cloud computing concept. All of the above for the cloud calculation theory basis for forming.

\subsection{Characteristics of Cloud Computing}

Expansion is one of the characteristics of cloud computing, in fact, the comprehensive utilization of resources, can be seen as a resource pool, to provide a number of services to achieve optimal allocation of different resources. Cloud computing to promote the transfer and interaction between the network management information, to achieve the goal of information sharing, to become the future of information technology resources, the key mode of construction. Cloud service platform can greatly improve work efficiency, create greater efficiency, reduce costs and expenses, and reduce the workload of staff. From either a digital information or computer resources in an arbitrary point of view, in the cloud platform, a single computer resources will get further integration, to achieve the optimal allocation, greatly improving the cost to construct the network service platform, can achieve remarkable results, the resources to make full use of. 


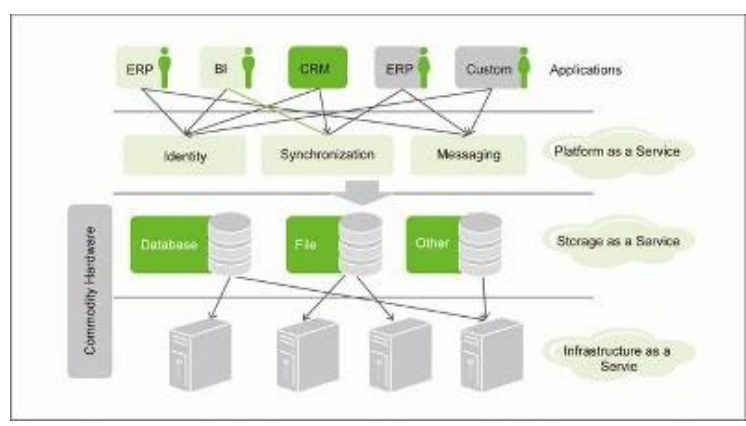

Fig. 1. Schematic diagram of cloud computing architecture

\subsection{Overview of Key Technologies in Cloud Computing}

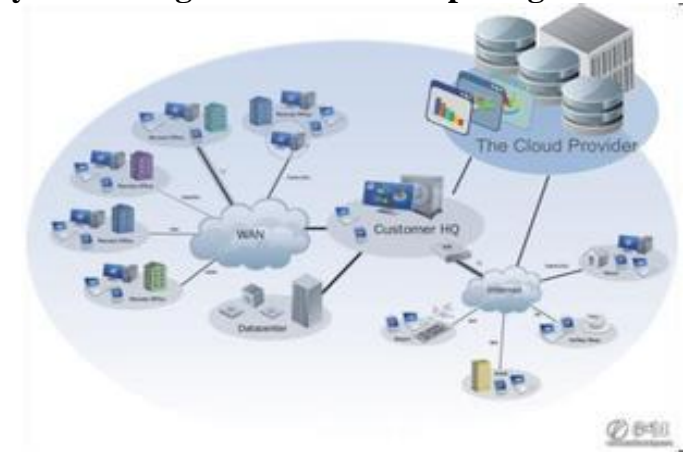

Fig. 2. Schematic diagram of key technologies of cloud computing

Network service platform based on cloud computing, play in the storage process are mainly rely on the form is distributed, confidence in a large number of data were multiple measures of protection, to improve the reliability of the system, to protect the security of data, will be more convenient services to users. Is the current cloud computing concept degree of perfection and related technology development status, Hadoop distributed file system and scalable distributed file system (Google GFS) discovery is a cloud platform used in the core technology of a storage area, in order to meet the large demand for cloud computing to the university sports educational administration teaching platform based on building management system to transfer application performance strong, large amount of service units of storage technology. GFS, distributed file system is the current two key cloud storage technology, which provides a basis for the study of cloud computing related Internet companies [3].

\subsection{Virtualization Technology Software Application}

Cloud computing platform in the basic equipment can work in a virtualized environment, will provide services to the user, through the role of virtualization technology, cloud platform to realize elastic telescopic, give full play to the software and hardware equipment, reduce the cost consumed in the configuration of infrastructure, improve the operation efficiency of the system is of great significance. The virtualization technology based on network cloud occupies a key position in the system program server, operating system and the central processing unit, 
which can improve the quality of network service. Depending on the resources of the computer set pattern can be virtual technology into polymerization, split into two categories, play a role in security network software and hardware resources independent of that cloud computing in the crack patterns of virtualization technology mainly refers to is further division of resources, the single resource into a plurality of resources of small units, but also in the real environment of different network resources integration [4].

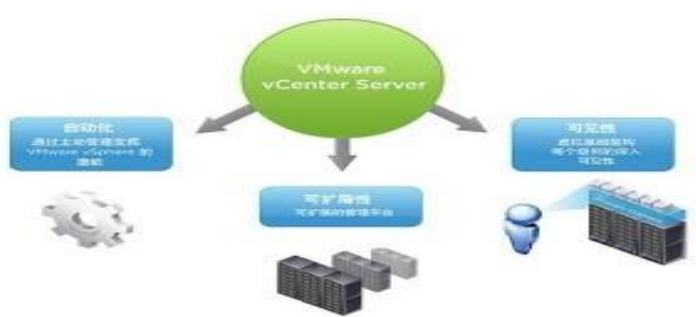

Fig. 3. Sketch map of virtualization technology

\section{Design and Implementation of the System}

Starting from the angle of sports educational management requirements, cloud services platform mainly depends on the application and data server to play a role, by the six servers constitute, which play a data backup and protect the function of the server are four, and the rest of the server is master data base of redundancy backup. IBM System x3950 M2 using cloud services platform in the educational management system, involving users equipped with scale Xpander option kit series products and added another four units system x3950 m2, makes the original management system of the server effectively expanded, from and more comprehensive adjusting information technology energy input, full and effective play cloud computing ability.

The cloud platform used in storage field equipment selection is Oceanspace S5000 storage system, the product in computing ability and data security, redundancy and has obvious advantages, in storage research occupies a very high position, can effectively deal with the huge data information, interface design is more flexible, data migration or backup services both is enhances the essence of the teaching quality, and plays a decisive role in the efficient operation of the system. The special disk repair and protection function in the storage function can ensure the availability of the data information stored in the disk, so as to avoid the occurrence of the abnormal condition, so that the whole system can be operated more reliably. In addition, in the advanced intelligent hard disk delay and power technology, can effectively reduce CPU energy consumption, to ensure the normal play of the heat dissipation function of the equipment and can reduce the fan operation, and alternating current consumption brought about by the energy waste due to. Compatible with existing network systems. Network system based on cloud platform of colleges and universities sports educational management system, in the design stage must be on the application to the hardware equipment to carefully review and analysis, ensuring the quality of the equipment meet the requirements, but also can ensure the reliability of the data information, management 
staff more convenient, high-efficient management, meet the users do not need to achieve personalized extended target, application efficiency to achieve the best[5].

\section{Conclusion}

This research project follow the idea of cloud computing, reconstruction and improvement of the educational management system of university sports performance and service level, through the system of data center and analysis, calculation, storage function, for users of the service customization, form the intelligent teaching provided a cloud service platform for information management. Student achievement management module, system management module, curriculum setting module, teaching module, student management module, database module of teaching resources, teaching quality assessing the management module together constitute the academic cloud. Which bear on teaching parameters setting, system user authority management, system maintenance and upgrade management, code set, data information of the unified processing module is the system management module, the module of the whole system of normal operation plays a key role. In the cloud computing concept gradually improve, cloud computing technology to obtain the development by leaps and bounds, built in the cloud computing based on Web services platform momentum of rapid development, has attracted wide attention of the society from all walks of life, provided the impetus for the future development of network services, but also in a certain extent promote the downstream product market development, stimulate business of research and development initiative, to make a greater contribution to further improvement of the computer information industry. In cloud computing platform for network service function, can make the software and hardware resources for centralized treatment can not only communication capability to ensure the service platform, to cloud the operation to provide security, significant advantages in terms of storage and computing functions. Established on the cloud platform based on Colleges and universities sports educational management system, design and implementation of the entire process follows the idea of cloud computing, make full use of the advantage of modern information technology, computer network, improve the teaching efficiency, provide the technique guarantee for the sustainable development of colleges and universities. The use of cloud computing, the teaching resources of colleges and universities of the exchange and sharing into reality, and has strengthened the contact between the universities, the sports teaching information updating, transmission, can let students and teachers in the teaching process form a more harmonious relationship in a short period of time.

\section{References}

[1] Jiang Daoxia, Fu Wei, Tu Gang, Li Jian, Xiong Ping. The design and implementation of university scientific research information system based on. NET Journal of Langfang Teacher's College (NATURAL SCIENCE EDITION). No. 06. (2013)

[2] Su chin. Research on the management system of University Library under the cloud computing. computer CD software and application. No. 20. (2012)

[3] Wang Lei, Zhang Tao. Design and implementation of the distance education system based on cloud computing in Colleges and universities. continuing education research. No. 07. (2011) 
[4] Yang Xufeng, Liao Shujian. Design and implementation of. fighting in Shanxi province sports scientific research management information system (Sports Forum). No. 01. (2011)

[5] Chen Yang, the high design and implementation of. Fight Tiemin. University sports information management system based on UML (Sports Forum). No. 03. (2010) 